\title{
Gastroduodenal ulceration after selective internal radiation therapy of liver tumor
}

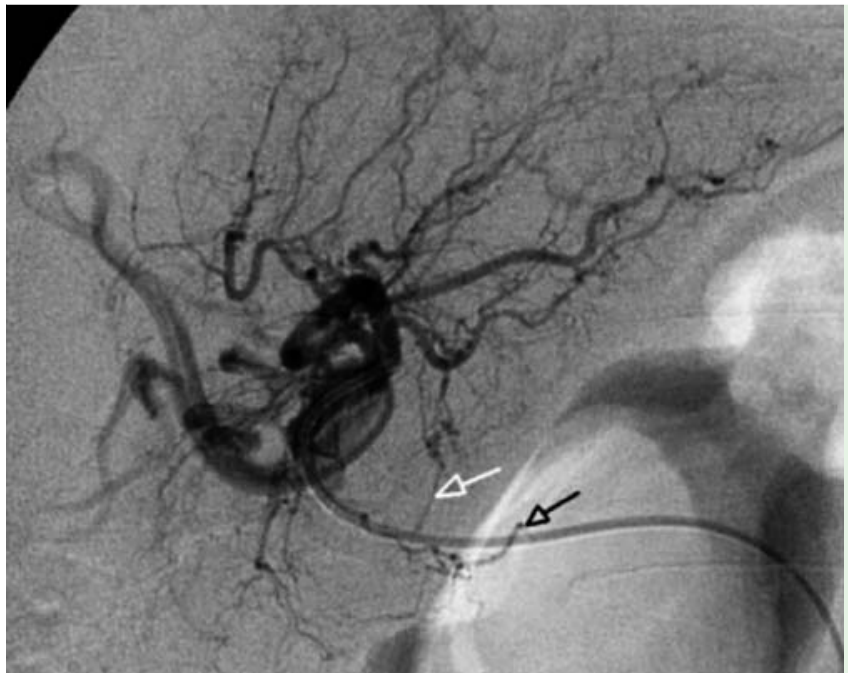

Fig. 1 Angiographic view in a 54-year-old female patient with hepatic metastases from breast cancer showing the hepatic arteries with aberrant branches of the left (white arrow) and right hepatic (black arrow) arteries extending to the stomach and not suitable for coiling. The injection site was distal to the aberrant branches to prevent reflux.

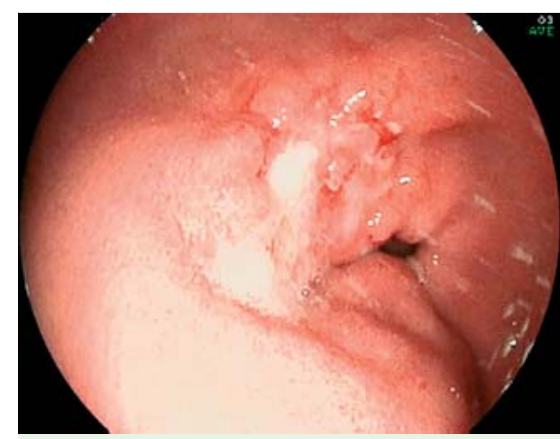

Fig. 2 Endoscopic image of the antral ulceration and severe mucosal inflammation in the lesser curve of the stomach 2 months after selective internal radiation therapy (SIRT).

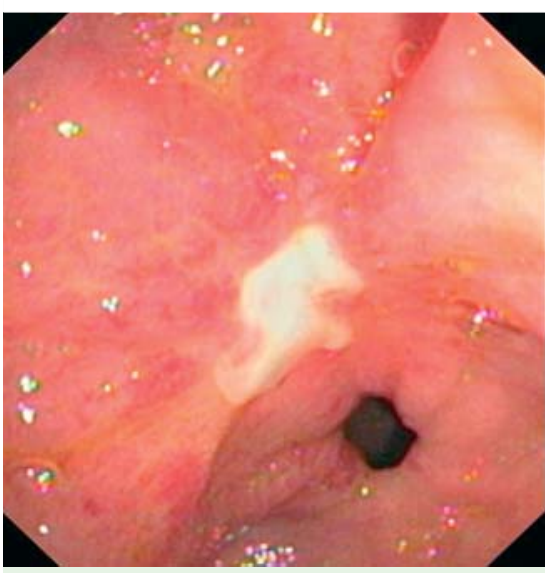

Fig. 3 Endoscopic image of the unchanged antral ulceration and severe mucosal inflammation in the lesser curve after 2 months of proton pump inhibitor therapy and 4 months after selective internal radiation therapy (SIRT).
Image-guided interstitial brachytherapy is used for the treatment of primary and secondary liver tumors. Millions of nonbiodegradable yttrium-90 ( $\left.{ }^{90} \mathrm{Y}\right)$-imprinted microspheres with a diameter of 29 $35 \mu \mathrm{m}$ are selectively infused by a catheter into the branch of the hepatic artery supplying the tumor. Thereby, a radiation dose of 30-60Gy can be applied to the tumor tissue. ${ }^{90} \mathrm{Y}$ is a beta-emitter with an average tissue penetration depth of approximately $2.5 \mathrm{~mm}$ and a physical half-life of 2.67 days (64.2 hours) [1,2]. Gastroduodenal ulceration occurs in less than $5 \%$ after selective internal radiation therapy (SIRT) [2-4].

We present a case of radiation-induced gastric ulceration after SIRT for the treatment of hepatic metastases from breast cancer in a 54-year-old female patient ( Fig. 1). At 2 months after SIRT, the patient presented with epigastric pain and was diagnosed as having anemia. Upper gastrointestinal endoscopy revealed a single, flat ulcer in the gastric antrum ( $\bullet$ Fig.2) and histological examination of two forceps biopsy specimens demonstrated chemical-reactive inflammation and ulceration without Helicobacter infection. Proton pump inhibitor (PPI) was prescribed as an oral medication.

A repeat endoscopy after 2 months revealed the ulceration was unchanged ( Fig.3). Upper endoscopic ultrasound showed the antral ulcer, but no tumor of the stomach ( $\bullet$ Fig. 4 ). At the same time, nearly 4 months after SIRT, 16 forceps biopsy samples were taken and histological examination revealed microspheres in the gastric tissue, with inflammation and ulceration ( $\bullet$ Fig.5). Retrospectively, the ulcer was caused by reflux of ${ }^{90} \mathrm{Y}$-micropheres into aberrant branches of the left and right hepatic arteries extending to the stomach ( Fig.1). Because of the presence of severe anemia, the patient underwent distal gastric resection. SIRTassociated gastrointestinal side effects are mainly diagnosed with the help of biopsies revealing pathognomonic microspheres. Endoscopists and pathologists should be aware of the findings in connection with this new therapy as well as its complications.

Endoscopy_UCTN_Code_CCL_1AB_2AD_3AC

Competing interests: None

I. Wallstabe ${ }^{1}$, G. Bakos ${ }^{2}$, A. Plötner², A. Kretzschmar ${ }^{3}$, D. Wegner ${ }^{4}$, K. Hohdorf $^{5}$, J. Scheibe ${ }^{6}$

${ }^{1}$ Department of Gastroenterology and Hepatology, Klinikum St Georg, Delitzscher Straße, Leipzig, Germany

${ }^{2}$ Department of Pathology und Tumor Diagnostics, Klinikum St Georg, Delitzscher Straße, Leipzig, Germany

${ }^{3}$ Department of Oncology und Haematology, Klinikum St Georg, Delitzscher Straße, Leipzig, Germany

${ }^{4}$ Specialist Practice in Gastroenterology, Leipzig, Germany

${ }^{5}$ Specialist Practice in Nuclear Medicine, Leipzig, Germany

${ }^{6}$ Department of Diagnostic and Interventional Radiology, Klinikum St Georg, Delitzscher Straße, Leipzig, Germany 


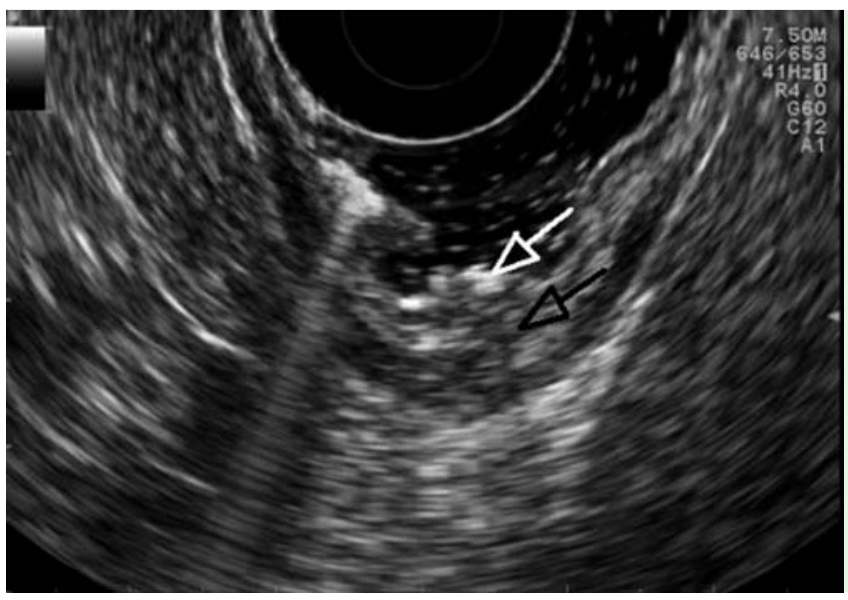

Fig. 4 Endosonographic image of the antral ulceration with a hyperechogenic line on the ulcer base (white arrow) and hypoechogenic tissue in the thickened submucosal layer (black arrow) 4 months after selective internal radiation therapy (SIRT).

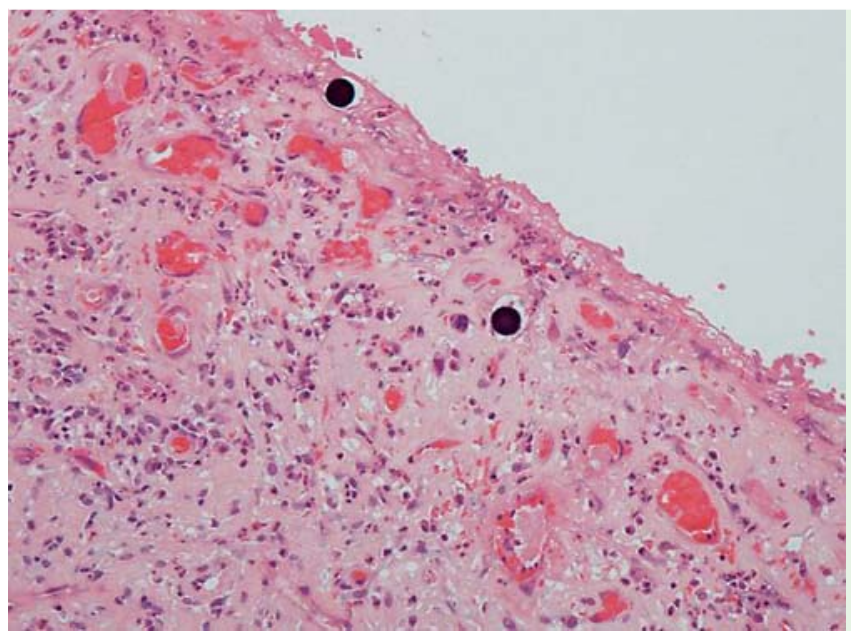

Fig. 5 Biopsy showing two round black ${ }^{90} Y$-microspheres within the capillaries at the ulcer base and associated regenerative mucosal changes of chemical-reactive type (hematoxylin and eosin stain).

\section{References}

1 Lau WY, Ho S, Leung TW et al. Selective internal radiation therapy for nonresectable hepatocellular carcinoma with intraarterial infusion of 90yttrium microspheres. Int J Radiat Oncol Biol Phys 1998; 40: 583-592

2 Prompers L, Bucerius J, Brans B et al. Selective internal radiation therapy (SIRT) in primary or secondary liver cancer. Methods 2011; 55: $253-257$

3 Konda A, Savin MA, Cappell MS et al. Radiation microsphere-induced GI ulcers after selective internal radiation therapy for hepatic tumors: an underrecognized clinical Entity. Gastrointest Endosc 2009; 70: 561 567

4 Ogawa F, Mino-Kenudson M, Shimizu M et al. Gastroduodenitis associated with yttrium 90-microsphere selective internal radiation: An iatrogenic complication in need of recognition. Arch Pathol Lab Med 2008; 132: $1734-1738$

\section{Bibliography}

DOI http://dx.doi.org/

10.1055/s-0032-1310023

Endoscopy 2012; 44: E354-E355

(c) Georg Thieme Verlag KG

Stuttgart · New York

ISSN 0013-726X

\section{Corresponding author}

\section{Wallstabe, MD}

Department of Gastroenterology and Hepatology Klinikum St. Georg

Delitzscher Str. 141

04129 Leipzig

Germany

Fax: +49-341-9092673

wallstabe@endoskopieren.de 